EPJ manuscript No.

(will be inserted by the editor)

\title{
Tau Identification at the Tevatron
}

\author{
Stephen Levy (on behalf of the CDF and D $\emptyset$ collaborations) \\ University of Chicago, Enrico Fermi Institute \\ Chicago, IL 60637
}

Received: date / Revised version: date

\begin{abstract}
Methods for reconstructing and identifying the hadronic decays of tau leptons with the CDF and $\mathrm{D} \emptyset$ detectors at the Fermilab Tevatron collider in Run II are described. Precision electroweak measurements of $W$ and $Z$ gauge boson cross sections are presented as well as results of searches for physics beyond the Standard Model with hadronically decaying tau leptons in the final state.
\end{abstract}

PACS. PACS-key discribing text of that key - PACS-key discribing text of that key

\section{Introduction}

The ability to reconstruct and identify tau leptons at the Tevatron is useful for making precise tests of the standard model in the electroweak sector as well as for probing for phenomena beyond the Standard Model (SM) of particle physics. The heavy mass of the tau relative to electrons and muons makes it an interesting candidate to study in the context of electroweak symmetry breaking. Historically, measurements of the tau have been useful in determining the agreement of lepton universality, and the value of the QCD coupling constant at low mass. In addition, efficiently reconstructing taus leads to a larger sample of top quarks which play an important role in the Tevatron's Run II goal of constraining the Higgs boson mass.

Additional motivation for studying tau leptons comes from the minimal supersymmetric extension of the standard model (MSSM) which provides an elegant solution to the problem of fine tuning of the Higgs mass. There are three neutral and two charged Higgs bosons in this model whose couplings to the tau are enhanced in various regions of the model parameter space. Searches for anomalous tau production at the Tevatron are useful in constraining new physics models.

This paper describes the methods used by the CDF and $\mathrm{D} \emptyset$ experiments to reconstruct and identify hadronic tau decays. The following sections describe the basic idea underlying the method at both experiments, the specific differences (most importantly the use of a neural net at $\mathrm{D} \emptyset$ ), the triggers used to collect samples of tau decays, results of $W$ and $Z$ cross sections where the boson decays to one or more tau leptons, and results of searches for physics beyond the SM with tau leptons in the final state.

\section{Hadronic Tau Reconstruction}

This section describes the reconstruction of hadronic tau decays at $\mathrm{CDF}$ and $\mathrm{D} \emptyset$. The branching fraction for hadronic tau decays is $\sim 65 \%$, with the most abundant final state consisting of exactly one charged pion and $\geq 0 \pi^{0} \mathrm{~s}$, referred to as one-prong decays. Reconstructing $\pi^{0} \mathrm{~s}$ is an important step in tau reconstruction since roughly threefourths of the one-prong decays contain at least one $\pi^{0}$. Reconstruction of leptonically decaying taus is accomplished via electron and muon identification and is not the subject of this proceeding.

Typically at the Tevatron identifying a lepton means identifying an isolated lepton and this distinction is paramount for taus. Hadronically decaying taus are essentially a narrow jet in the detector consisting of charged track(s) pointing to hadronic calorimeter energy deposition and potentially associated electromagnetic energy from $\pi^{0} \rightarrow \gamma \gamma$ decays.

The difficulty of reconstructing taus in a hadron collider environment, of course, stems from the fact that some fraction of jets and electrons are also "narrow jets". The ratio of QCD jet production to the electroweak cross section scale is order one million. Though jets may consist of the same final state of charged and neutral particles as taus, they are not an irreducible background since the final state that results from a tau decay will have an invariant mass less than the tau mass and events containing a tau will contain missing energy due to the presence of the tau neutrino. Additionally, the tau travels $\sim 100 \mu \mathrm{m}$ before decaying which means that its decay products will have larger impact parameters (with respect to the event primary vertex) that can be measured using silicon detectors.

However, isolation provides the most powerful variable for distinguishing hadronically decaying taus from jets. Tau identification at $\mathrm{CDF}$ and $\mathrm{D} \emptyset$ begins by requir- 
ing $\sim 5 \mathrm{GeV}$ of energy deposited in a narrow region of the calorimeter with a well measured track pointing at the cluster. Narrow is dictated by the segmentation of the calorimeter, which is sufficiently more granular at $\mathrm{D} \emptyset$. Full details of the detectors at each experiment detectors are given elsewhere $[1,2]$. The specifics of the tau reconstruction diverge at this point and are described for each experiment in the following sections.

\subsection{Tau Identification at CDF}

Tau reconstruction begins at $\mathrm{CDF}$ with a well reconstructed track, termed the seed track, pointing at a narrow calorimeter cluster $(|\eta|<1)^{1}$ which consists of $\leq 6$ towers. A signal cone is defined with respect to the direction of a seed track $\left(p_{T}>6 \mathrm{GeV} / c\right)$ whose opening angle is inversely proportional to the calorimeter energy of the cluster. At high calorimeter energy the angle is fixed to a minimum of $50 \mathrm{mrad}$ due to resolution and at low energy to $175 \mathrm{mrad}$. An isolation region is defined as the annulus between the signal cone and 30 degrees as shown in Fig. 1. Candidates are rejected if the isolation region contains well measured tracks. The total number of well measured tracks within the single cone is commonly referred to as the number of prongs of the decay (the number of two-pronged taus is useful in understanding the number of fake candidates).

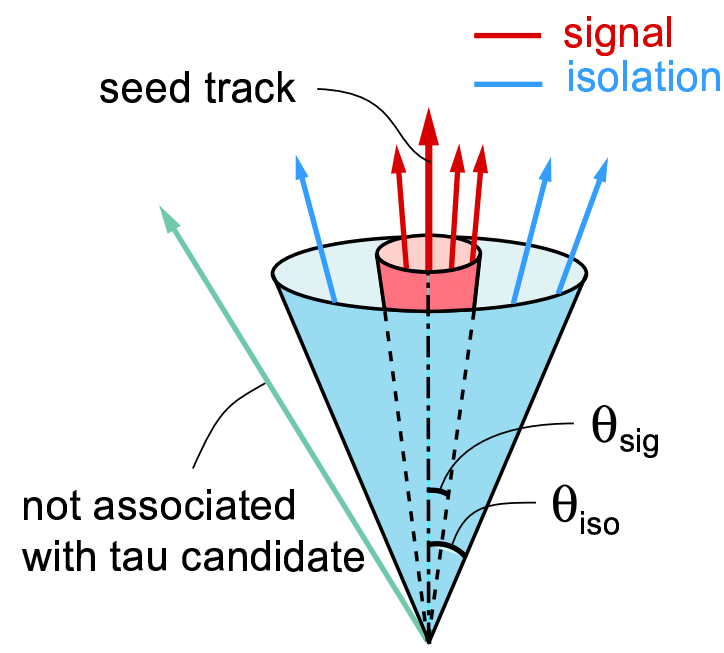

Fig. 1. Schematic illustration of the energy dependent signal and isolation region used to define a tau candidate at CDF.

The electromagnetic (EM) calorimeter at CDF has a multi-wire proportional chamber (CES) embedded at approximately six radiation lengths that is used to reconstruct $\pi^{0}$ candidates (as well as electrons and photons) with energy $>500 \mathrm{MeV}$. The CES provides two orthogonal measurements of the position of the $\pi^{0}$ candidate with spatial resolution of $\sim 3 \mathrm{~mm}$ which are matched based on their consistency in terms of deposited energy. The energy

\footnotetext{
1 The pseudo-rapidity $\eta$ is defined as $-\ln (\tan (\theta / 2))$ where $\theta$ is the polar angle with respect to the proton beam direction
}

assigned to a single $\pi^{0}$ candidate is the energy measured in the electromagnetic calorimeter after an average correction is made for energy deposited by charged tracks in the tower, typically of order of a few $\mathrm{GeV}$. For multiple $\pi^{0}$ candidates, the energy in the EM calorimeter is apportioned according to the CES energy of each candidate. Additionally, a variable sized signal cone whose opening angle is inversely to the candidate calorimeter energy is defined for the $\pi^{0} \mathrm{~s}$ and candidates are rejected if there are well measured $\pi^{0} \mathrm{~s}$ in the annulus between the signal and isolation cones. The tau candidate four momentum is constructed from the sum of the four momenta of the tracks and $\pi^{0} \mathrm{~s}$ in the signal region.

Tau identification typically refers to extra requirements that are applied to the reconstructed tau candidates. The specific requirements can vary based on the analysis but typical ones are summarized. Taus are required to have a mass consistent with a hadronic tau decay $\left(<1.8 \mathrm{GeV} / c^{2}\right)$. Also, to discriminate taus from electrons, the variable $\xi$ is defined as

$$
\xi=E_{T}^{H A D} / \sum\left|\boldsymbol{p}_{t r k}\right|
$$

where $E_{T}^{H A D}$ is the transverse component of the energy that the tau candidate deposited in the hadronic calorimeter. Requiring that $\xi>0.2$ substantially reduces the number of electrons that are reconstructed as tau candidates. Additionally, tau candidates are required to consist of one or three prongs with the absolute value of the sum of the charge of the tau tracks equal to one. The efficiency to reconstruct and identify simulated tau decays as a function of the true energy of the hadronic system is shown in Fig. 2. The efficiency plateaus around $45 \%$ above $50 \mathrm{GeV}$. The probability for a jet to be reconstructed as a tau candidate, termed fake rate, is measured in data events triggered by jets with various energy thresholds. The fake rate is parameterized in terms of the jet cluster energy and the ratio of the jet energy and mass. Fig. 3 shows the rate of jets misidentified as taus as a function of jet energy for jets passing a $50 \mathrm{GeV}$ trigger requirement. The fake rate is $\sim 0.5 \%$ at $50 \mathrm{GeV}$.

\subsection{Tau Identification at $\mathrm{D} \emptyset$}

A similar method for reconstructing hadronic tau decays is employed at $\mathrm{D} \emptyset$ but the tau identification method differs from that used at CDF. The tau candidates are found by matching a track with $p_{T}>1.5 \mathrm{GeV}$ to a narrow calorimeter cluster with $E_{T}>5 \mathrm{GeV}$ [5]. In this case, narrowness is defined by the $E_{T}$ weighted spread in the cluster width based on the spatial separation of the tower center and tau cluster center. Additional tracks within a cone $R=\sqrt{(\Delta \phi)^{2}+(\Delta \eta)^{2}}<0.3$ of the calorimeter cluster are added if the invariant mass of the resulting candidate calculated from the tracks is consistent with a tau. Subclusters with minimum energy $800 \mathrm{MeV}$ are constructed from the cells in the EM section of the calorimeter as $\pi^{0}$ candidates. The tau candidates are separated into three classes based on the tracking and calorimetry information: (1) single track with no $\pi^{0}$ candidates, (2) 


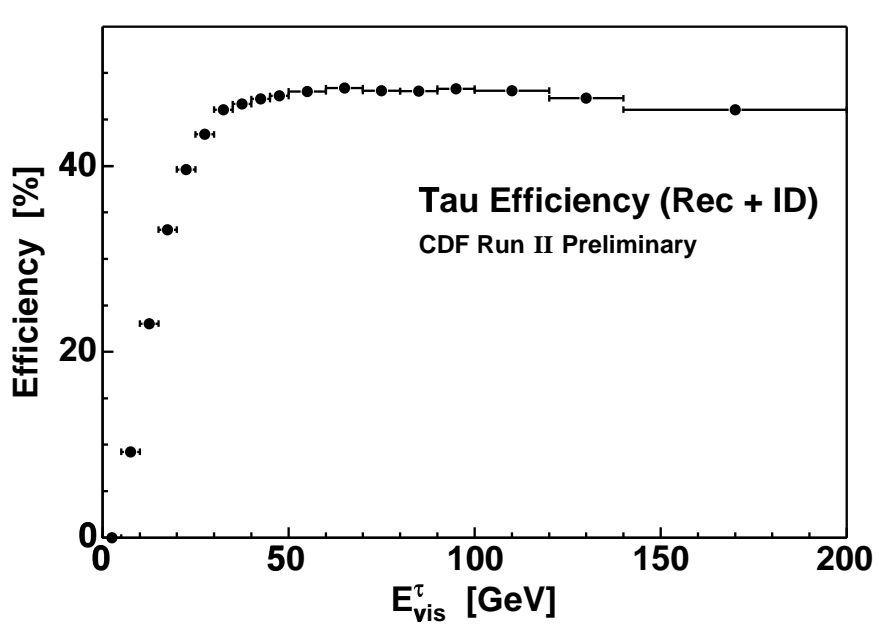

Fig. 2. Tau reconstruction and identification efficiency at CDF for simulated hadronically decaying taus as a function of visible tau energy.

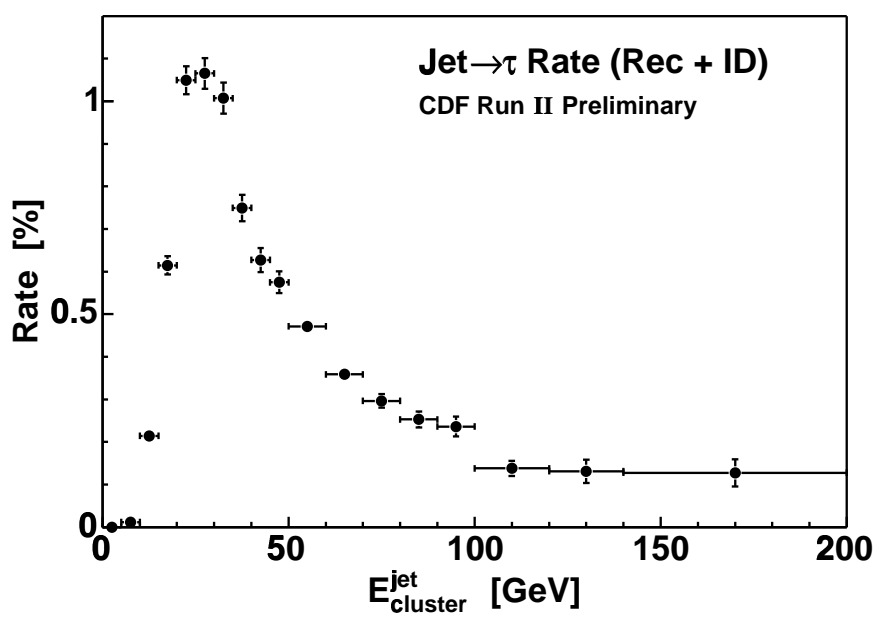

Fig. 3. Rate of jets misidentified as taus as function of jet energy at CDF.

single track with $\geq 1 \pi^{0}$ candidate, or (3) more than one associated track.

A neural network $(\mathrm{NN})$ is used to separate these tau candidates from the large background of jets. The neural network consists of a single input layer with several nodes, a single hidden layer, and a single output layer. Separate NN training is performed for each tau category described above using Monte Carlo simulation of single tau leptons for the single and jets from data events for the background. The input variables of the neural net [5] are typically ratios of the tau candidate kinematic properties to minimize the dependence on the absolute energy scale of the simulation. For example, there is a powerful profile variable defined as $\left(E_{T_{1}}+E_{T_{2}}\right) / E_{T}^{\tau}$ where $E_{T_{1}}$ and $E_{T_{2}}$ are the transverse energies of the two most energetic calorimeter towers in the tau cluster $\left(E_{T}^{\tau}\right)$. This variable is used in the $\mathrm{NN}$ for all tau types but others are specific to the tau candidate class. It is important to note that the training is not the same for all $\mathrm{D} \emptyset$ tau analyses: the train-
Table 1. Efficiency and fake rate for tau candidates in $Z \rightarrow \tau \tau$ simulation and QCD jets in data respectively using D $\emptyset$ 's neural net.

\begin{tabular}{lll}
\hline & $\begin{array}{l}\text { Efficiency } \\
(Z \rightarrow \tau \tau)\end{array}$ & $\begin{array}{l}\text { Fake Rate } \\
(\mathrm{QCD})\end{array}$ \\
\hline type1 & $0.78 \pm 0.03$ & $0.145 \pm 0.014$ \\
type2 & $0.75 \pm 0.02$ & $0.042 \pm 0.004$ \\
type3 & $0.73 \pm 0.02$ & $0.039 \pm 0.002$ \\
\hline
\end{tabular}

ing may rely on event information for the physics being investigated. The efficiency for taus selected in $Z \rightarrow \tau \tau$ Monte Carlo simulation after a cut on the output of the NN is shown in Table 1 along with the rate that QCD jets in data are identified as taus for each tau class [4]. Relative to the selection used by CDF, D $\emptyset$ has a larger efficiency with a correspondingly larger fake rate. There are additional restrictions that are used in tau identification that are analysis dependent: some apply an anti-muon requirement on the tau candidate or use an additional NN to separate tau from electron candidates. Performance of the $\mathrm{NN}$ in data will be presented in the context of a $Z \rightarrow \tau \tau$ analysis described in Sec. 4.1.

\section{Tau Triggers}

Before presenting the results of physics analyses relying on tau lepton reconstruction, it is necessary to briefly review the method by which both experiments collect large samples of tau decays. Both experiments have a three level trigger system which is designed to reduce the nominal crossing rate of $7.6 \mathrm{MHz}$ to approximately $50 \mathrm{~Hz}$ which can be written to tape. The trigger consists of hardware at Levels 1 and 2 (using only axial tracking information) and a system of software algorithms executed on a computer farm at Level 3. The CDF tau triggers [3] search for a tau candidate combined with large missing transverse energy or another tau candidate, and of lepton+track triggers which are used to identify an electron or muon in combination with an isolated track. $\mathrm{D} \emptyset$ uses their $\mathrm{NN}$ to identify low $p_{T}$ tau candidates at Level 3. Many D $\emptyset$ tau analyses currently rely on the presence of a muon or electron in the event which forms the basis for the trigger. A typical rate of the electron (muon) +track trigger at CDF is $3.0(1.5) \mathrm{Hz}$ at Level 3 for an instantaneous luminosity of $1 \mathrm{e} 32 / \mathrm{cm}^{2} / \mathrm{s}$.

\section{Electroweak Tau Results}

Both CDF and $\mathrm{D} \emptyset$ have demonstrated the ability to reconstruct large samples of hadronically decaying taus in electroweak measurements of gauge boson cross sections.

\subsection{D $\emptyset$ Electroweak Tau Results}

$\mathrm{D} \emptyset$ has measured the cross section for $Z$ production times the branching fraction to tau leptons in the channel in 
which one tau decays leptonically into $\mu \nu_{\mu} \nu_{\tau}$ and the other into hadrons $+\nu_{\tau}$ [5]. The analysis is based on $226 \mathrm{pb}^{-1}$ of data. The event selection basically consists of finding an isolated muon $p_{T}>12 \mathrm{GeV} / c$ opposite a tau candidate. The events with muon and tau candidates with the same charge are used to estimate the background from QCD (primarily $b \bar{b}$ ) and the additional background from $W \rightarrow \mu \nu+$ jets was estimated in magnitude and shape from Monte Carlo simulation. By requiring that the NN output for the tau candidate be $>0.8$ the signal to background ratio is improved by a factor of $\sim 1200$ to roughly $1: 1$. Fig 4 compares the expected distributions for the tau $E_{T}$ and muon $p_{T}$ (after the NN cut) to the background from data and to the data after the background has been subtracted. With a signal sample of $\sim 900$ events $\mathrm{D} \emptyset$ measures the product of the $Z$ cross section times the branching fraction to tau leptons that is in good agreement with the NNLO prediction of $242 \pm 10 \mathrm{pb}$.
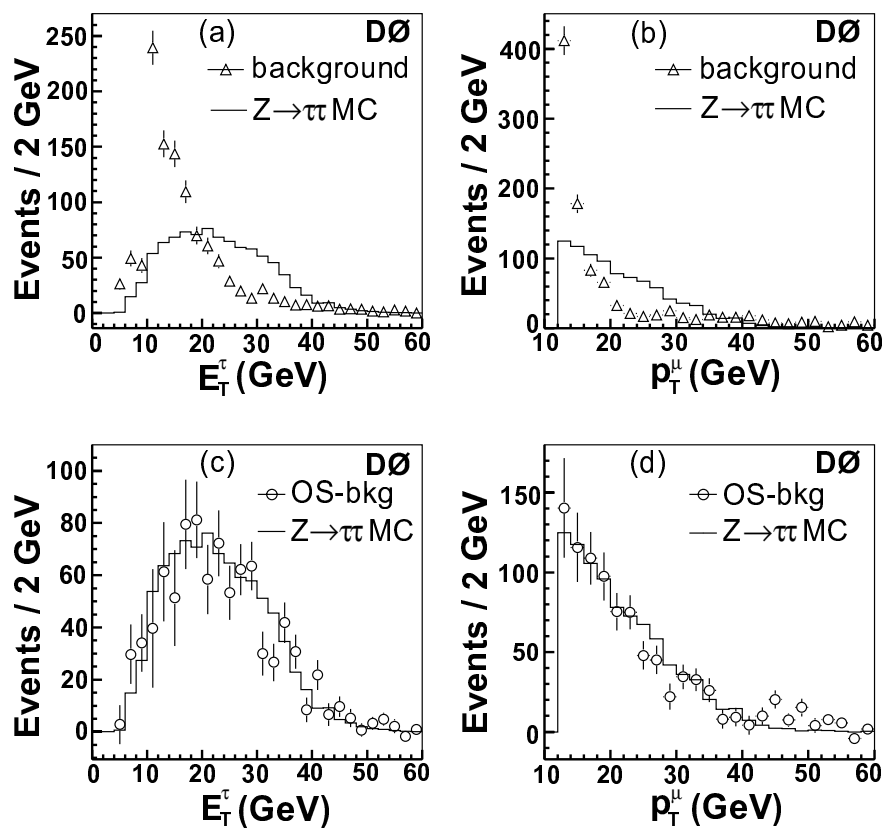

Fig. 4. $\mathrm{D} \emptyset$ comparison of tau $E_{T}$ and muon $p_{T}$ distributions after NN cut; (a), (b) estimated background (open triangles) and predicted $Z \rightarrow \tau \tau$ signal (histogram); (c), (d) background subtracted data (open circles) and predicted $Z \rightarrow \tau \tau$ signal.

\subsection{CDF Electroweak Tau Results}

CDF has measured the product of the cross section for $W$ production times the branching fraction for $W \rightarrow \tau \nu$ using $72 \mathrm{pb}^{-1}$ of data [6]. The event selection requires large missing transverse energy $(>25 \mathrm{GeV})$ and a tau candidate without other significant jet activity. This selection results in an abundant pure sample of hadronic taus that are useful for understanding the differences between the tau reconstruction in data and Monte Carlo simulation. The signal to background ratio for these events is $\sim 3$ with a yield of 24 events $\mathrm{pb}^{-1}$. Fig. 5 shows the distribution of the number tracks in the tau candidates along with the expected background. The analysis additionally measures the ratio of branching fractions for $W \rightarrow \tau \nu$ and $W \rightarrow e \nu$ and finds that the ratio of the tau and electron coupling constants to the $W$ are consistent with $4 \%$ precision.

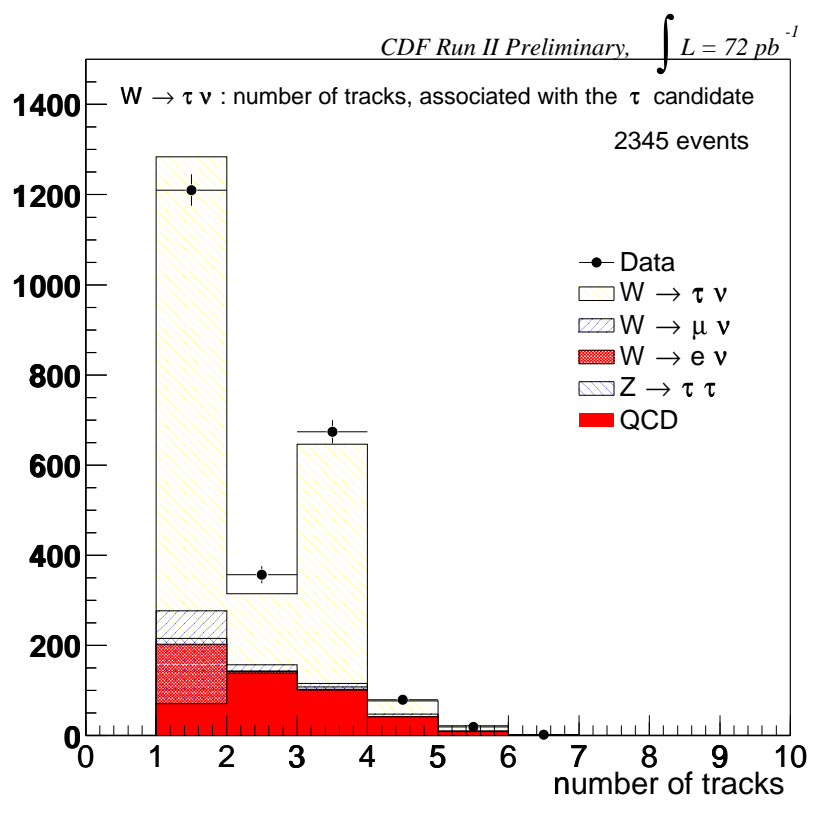

Fig. 5. CDF charged multiplicity distribution for $W \rightarrow \tau \nu$ candidates.

CDF has also measured the cross section for $Z$ production times the branching fraction for $Z \rightarrow \tau \tau$ in events where one tau decays hadronically and the other decays to $e \nu_{e} \nu_{\tau}$ with $72 \mathrm{pb}^{-1}$ of data [6]. The result is consistent with SM expectations.

\section{Searches for New Physics}

With the tau electroweak precision measurements in hand, both experiments are focusing efforts on searches for physics beyond the SM that include taus in the final state. $D \emptyset$ has a preliminary conference result involving chargino and neutralino searches in the $e \tau \ell$ final state, as well as for R-parity violated supersymmetry in the eer final state. CDF has a preliminary conference result for a search for pair production of supersymmetric top quarks decaying via $R$-parity violating coupling to b-quark and a tau lepton. Also, CDF has published the results of a search for anomalous resonant production of tau lepton pairs with large invariant mass [7] and submitted for publication a search a for neutral MSSM Higgs boson decaying to tau pairs [8]. 


\section{Conclusion}

Though the study of final states with tau leptons is difficult in hadron environments, both CDF and $D \emptyset$ have demonstrated the ability to collect, reconstruct and identify large samples of tau decays. The probability for a jet to be identified as a tau is well measured using data. These samples have been used to measure electroweak gauge boson cross sections which are consistent with SM expectations. The Tevatron experiments are ramping up their searches for anomalous production of tau decays that would help constrain physics beyond the SM.

\subsection{Acknowledgments}

I would like to thank the HCP organizers for providing an excellent conference as well as the CDF and $\mathrm{D} \emptyset$ tau groups for their thoughtful contributions to this proceeding.

\section{References}

1. D. Acosta et al. (CDF Collaboration), Phys. Rev. D 71, 032001 (2005).

2. V. Abazov et al. (D $\emptyset$ Collaboration), Nucl. Instrum. Methods Phys. Rev. A, in preparation for submission.

3. S. Baroiant et al. (CDF Collaboration), Nucl. Instrum. Methods Phys. Rev. A518, 249 (2004).

4. A. Le Bihan et al. (D $\emptyset$ Collaboration), Nucl. Phys. Proc. Suppl 144, 333-340 (2005).

5. V. Abazov et al. (D $\emptyset$ Collaboration), Phys. Rev. D 71, 072004 (2005)

6. A. Safonov et al. (CDF Collaboration), Nucl. Phys. Proc. Suppl 144, 323-332 (2005).

7. D. Acosta et al. (CDF Collaboration), Phys. Rev. Lett. 95, 131801 (2005).

8. A. Abulencia et al. (CDF Collaboration), submitted to PRL, hep-ex/0508051.

9. Author, Book title (Publisher, place year) page numbers 